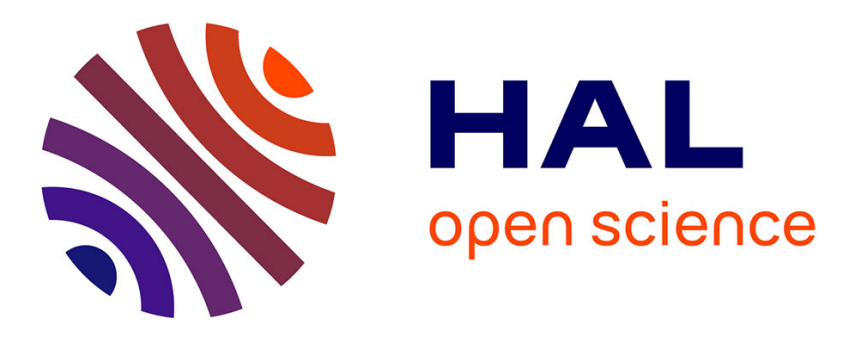

\title{
De la gestion des masses à une offre de formation individualisée en anglais-LANSAD : tensions et structuration
}

\author{
Linda Terrier, Cristelle Maury
}

\section{- To cite this version:}

Linda Terrier, Cristelle Maury. De la gestion des masses à une offre de formation individualisée en anglais-LANSAD : tensions et structuration. Recherche et pratiques pédagogiques en langues de spécialité - Cahiers de l'APLIUT, 2015, Vol. XXXIV No 1, pp.67-89. 10.4000/apliut.5029 . halshs02272486

\section{HAL Id: halshs-02272486 \\ https://shs.hal.science/halshs-02272486}

Submitted on 27 Aug 2019

HAL is a multi-disciplinary open access archive for the deposit and dissemination of scientific research documents, whether they are published or not. The documents may come from teaching and research institutions in France or abroad, or from public or private research centers.
L'archive ouverte pluridisciplinaire HAL, est destinée au dépôt et à la diffusion de documents scientifiques de niveau recherche, publiés ou non, émanant des établissements d'enseignement et de recherche français ou étrangers, des laboratoires publics ou privés. 
Cahiers de l'Apliut

Vol. XXXIV No 1 | 2015

La formation en langues/LANSAD dans les centres de langues : état des lieux et perspectives

\section{De la gestion des masses à une offre de formation individualisée en anglais-LANSAD : tensions et structuration}

From managing masses to offering individualised learning in a language center: tensions in structuring the field of English for specialists of other domains

Linda Terrier et Cristelle Maury

\section{OpenEdition}

Journals

Édition électronique

URL : http://journals.openedition.org/apliut/5029

DOI : 10.4000/apliut.5029

ISSN : 2119-5242

Éditeur

APLIUT

Édition imprimée

Date de publication : 15 janvier 2015

Pagination : 67-89

ISBN : $978-2-8218-5355-3$

ISSN : 2257-5405

Référence électronique

Linda Terrier et Cristelle Maury, «De la gestion des masses à une offre de formation individualisée en anglais-LANSAD : tensions et structuration », Recherche et pratiques pédagogiques en langues de spécialité [En ligne], Vol. XXXIV Nº 1 | 2015, mis en ligne le 01 octobre 2016, consulté le 02 mai 2019. URL : http://journals.openedition.org/apliut/5029 ; DOI : 10.4000/apliut.5029 


\section{Linda Terrier et Cristelle Maury}

\section{Université Toulouse - Jean Jaurès}

De la gestion des masses à une offre de formation individualisée en anglaisLANSAD : tensions et structuration

\section{Mots-clés}

anglais de spécialité, auto-formation guidée, CRL, cours magistral, innovation, massification, politique linguistique, structuration du LANSAD

\section{Résumé}

L'enseignement des langues aux spécialistes d'autres disciplines s'est développé plus tardivement qu'ailleurs dans les universités ALLSHS, probablement parce qu'elles constituent les seuls lieux où la formation LANSAD cohabite avec la formation LEA et la formation pour spécialistes (LLCE). Or on assiste aujourd'hui à une massification du secteur LANSAD en ALLSHS au niveau national.

À partir de leur exemple local, les auteures déconstruisent ce phénomène de massification afin d'en comprendre les enjeux. Elles analysent la manière dont l'équipe pédagogique a proposé une offre de formation raisonnée organisée en deux pôles: l'un centré sur le Cadre européen commun de référence pour les langues, l'autre articulé autour d'un dispositif d'autoformation guidée en CRL.

Les auteures montrent les tensions entre questions politiques, structurelles, didactiques et pédagogiques, et mettent en évidence des mécanismes de structuration du secteur LANSAD qui pourraient être propres au domaine ALLSHS.
From managing masses to offering individualised learning in a language center: tensions in structuring the field of English for specialists of other domains

\section{Keywords}

ESP, guided self-learning, language centre, lecture, innovation, massification, language policy, structuring the field of LSP

\section{Abstract}

The teaching of English to specialists of other domains of study has developed much more slowly in French Faculties of Humanities than in others, such as Faculties of Science and Technology or law schools. Despite this late start however, the field of English for specialists of other domains in Faculties of Arts and Human Sciences is now strongly developing

Using a local situation as an example, the authors first deconstruct the phenomenon so as to understand exactly what is at stake in the massive increase in students learning ESP. They then analyse the way the teaching team has managed to build a curriculum that would be well-reasoned and yet adapted to the masses. This was done by organising the curriculum around two different tools: the CEFR and self-directed learning in a language centre. The analysis of this curriculum allows the authors to show the tensions between politics, equipment, didactics and pedagogy, and they underline some of the specific mechanisms involved in building curricula for teaching ESP in Faculties of Arts and Human Sciences. 


\section{De la gestion des masses à une offre de formation individualisée en anglais-LANSAD : tensions et structuration}

\section{Introduction}

À l'Université des arts, lettres, langues, sciences humaines et sociales (ALLSHS) de Toulouse 2 - Jean Jaurès (UT2J), la «politique des langues pour tous » décidée par la Présidence en 2010 a entraîné une massification de la filière LANSAD ${ }^{1}$ sans précédent. Cette politique des langues volontariste, en lien avec les politiques européennes, a coïncidé avec la démolition/reconstruction intégrale de l'Université ${ }^{2}$. C'est dans ce contexte que le «Centre de Ressources des Langues » (CRL) de l'UFR langues, lettres et civilisations étrangères où cohabitent les trois filières consacrées aux langues, LANSAD, LEA et LLCE ${ }^{3}$, a vu le jour en 2009.

Si elle n'en est pas la cause première, cette transformation physique de l'UFR LLCE et la création du CRL se sont accompagnées d'une évolution inédite de l'offre de formation en langues pour les étudiants spécialistes d'autres disciplines. L'effort de structuration de l'anglais-LANSAD par les enseignants et enseignantschercheurs du secteur a consisté, en particulier, à passer de la seule problématique de «gestion des masses » à un enseignement/apprentissage raisonné de la langueculture, notamment en intégrant le CRL à l'ensemble de l'offre de formation afin de conduire les étudiants vers l'autonomie d'apprentissage (Holec 1990) et vers l'autonomie langagière (Bailly $1998: 19$ ).

La description du contexte institutionnel dans la première partie de cet article nous permettra d'analyser les caractéristiques de la massification de la filière LANSAD dans le contexte spécifique d'une université ALLSHS. Nous dégagerons les tensions entre questions politiques, structurelles, didactiques et pédagogiques et tenterons de cerner les enjeux qui en découlent. Dans la seconde partie, nous montrerons comment l'équipe sur le terrain s'est réapproprié un processus de structuration de l'offre de type descendant pour passer d'une problématique de «gestion des masses» à une proposition de formation raisonnée autour des niveaux du $C E C R L^{4}$ pour les étudiants du L2 à M2, et de formation individualisée

\footnotetext{
${ }^{1}$ Voir glossaire des acronymes en annexe 1.

${ }^{2}<$ http://lareconstruction.univ-tlse2.fr/accueil-la-reconstruction/un-peu-d-histoire/les-dates-cles/>

${ }^{3}$ La filière LANSAD est consacrée aux étudiants spécialistes d'autres disciplines que les langues. La filière LEA vise à former des spécialistes de langues appliquées à l'économie. La filière LLCE est la filière pour spécialistes des langues, littératures et civilisations étrangères.

${ }^{4}$ Cadre européen commun de référence pour les langues (Conseil de l'Europe 2001).
} 
pour les étudiants de L1 au CRL. Au-delà des enjeux locaux, nous tenterons d'établir une liste de critères pertinents à la réflexion sur la structuration du LANSAD en ALLSHS afin de faire émerger des invariants pour la construction de l'objet «LANSAD » en contexte ALLSHS.

\section{Politique des langues et massification du secteur LANSAD à l'UT2J}

La structuration de la formation LANSAD à l'UT2J s'est opérée, à l'origine, par un processus « descendant» («top-down ») ou, selon les termes de Nicolas Guichon (2012: 2), par une «logique d'imposition» qui «émane des autorités sociopolitiques $[\ldots]$ et économiques $[\ldots]$ qui déploient des discours valorisants voire militants $»$.

Dans notre cas, le discours concerne la place des langues dans les formations de «non-spécialistes » de l'enseignement supérieur. Il émane des autorités sociopolitiques $^{5}$ qui se situent à trois niveaux: européen (politiques des langues européennes, processus de Bologne et stratégie de Lisbonne), national (place des langues pour le secteur LANSAD dans la réforme $\mathrm{LMD}^{6}$ ) et local (politiques des langues par la Présidence de l'Université). Il a donné lieu à une massification du secteur LANSAD dans une université où l'enseignement des langues était jusque-là majoritairement consacré aux spécialistes.

\subsection{Phase 1: une politique des langues pour les spécialistes d'autres disciplines}

Cette logique d'imposition est demeurée implicite à l'UT2J puisqu'aucun texte ne mentionne explicitement une "politique des langues». L'évolution de l'offre locale de formation en langues montre toutefois qu'une politique des langues a été organisée en deux phases par la Présidence de l'Université : de 2002 à 2009, et de 2010 à aujourd'hui.

\subsubsection{Politiques des langues européennes}

Gail Taillefer (2009 : 72) explique que la réforme LMD en France est une réponse nationale à la création de l'espace européen de l'enseignement supérieur, prévue par la signature de la déclaration de Bologne (1999) et dont l'objectif est de «favoriser la mobilité des étudiants, des enseignants et des chercheurs, l'employabilité, le plurilinguisme et le multiculturalisme ».

Cette réforme a encouragé une structuration plus formelle de l'offre de formation en langues dans l'enseignement supérieur français, en particulier parce qu'elle stipule la nécessité de valider « l'aptitude à maîtriser au moins une langue vivante

\footnotetext{
${ }^{5}$ Les autorités économiques mettent quant à elles une pression certaine sur la maîtrise de l'anglais, considéré comme langue de l'employabilité et de la réussite des entreprises.

${ }^{6}$ Organisation du cursus universitaire français en trois diplômes : Licence-Master-Doctorat.
} 
étrangère $»^{7}$ au niveau Master. Si l'arrêté de 2002 ne dit rien sur le sens à donner à la notion de "maîtrise » d'une langue étrangère (ni sur le sens "d'aptitude à maîtriser », pour le moins étrange), dès 2003, la commission des titres d'ingénieur ${ }^{8}$ se saisissait de cette question et proposait la validation du niveau B2 du CECRL comme prérequis à l'obtention du diplôme d'ingénieur. C'est également ce qui s'est passé à l'UT2J dès 2002 où le niveau B2 a été fixé comme l'objectif à atteindre en fin de formation.

\subsubsection{Frénésie du niveau $B 2$}

Le paradoxe d'un niveau B2 dans une langue étrangère en fin de formation dans l'enseignement supérieur a régulièrement été souligné puisque le même niveau B2 est visé à la sortie du lycée ! On peut donc émettre l'hypothèse que le niveau de « maîtrise » fixé dans l'arrêté de 2002 s'entend comme un niveau expressément « universitaire ». On différencierait alors un niveau B2 « universitaire » du niveau B2 « scolaire ».

L'existence de certifications ${ }^{9}$ visant l'insertion professionnelle et/ou l'intégration dans une université étrangère semble confirmer l'existence d'un niveau B2 universitaire, distinct d'un B2 scolaire. Déterminer les contours d'un tel niveau B2 universitaire, par exemple sur la base d'une maîtrise de la langue de spécialité liée au domaine de spécialisation des étudiants, permettrait non seulement d'orienter la structuration des formations LANSAD mais aussi de sortir du paradoxe d'un niveau visé identique à la sortie de lycée et, cinq ans plus tard, à la fin du M2.

La décision de la commission des titres d'ingénieur, reprise dans de nombreux autres établissements de l'enseignement supérieur, d'imposer un niveau B2 à la sortie des formations d'ingénieurs a, quoi qu'il en soit, engendré une « frénésie du niveau $\mathrm{B} 2 »^{10}$, et «la montée en force d'une logique de certification » (Rivens Mompean 2013 : 28), conséquences directes de la loi LMD.

\subsubsection{Principes fondateurs de la politique des langues locale}

La première phase de la politique des langues a ainsi été marquée par la mise en place du CLES et l'UT2J est devenue centre certificateur agréé pour l'ensemble du site toulousain en 2008. Cette première phase s'est aussi concrétisée par l'obligation, pour tous les étudiants du secteur LANSAD, de suivre deux modules de langue étrangère durant leur L2 (40h de langue par module), et s'est traduite par le recrutement en 2007, 2008 et 2009 de trois enseignants-chercheurs (MCF)

\footnotetext{
${ }^{7}$ Arrêté du 25 avril 2002 relatif au diplôme national de master (article 6).

${ }^{8}$ La commission des titres d'ingénieur est un organisme indépendant, chargé par la loi française depuis 1934 d'habiliter toutes les formations d'ingénieur (<http://www.cti-commission.fr/>).

${ }^{9}$ TOEIC, TOEFL, FCE, IELTS, CLES, etc.

${ }^{10}$ Expression proposée en écho à la « frénésie de la certification » mentionnée par Taillefer (2009: 71$)$.
} 
devant effectuer au moins $80 \%$ de leur service d'enseignement en LANSAD, et d'un enseignant du secondaire affecté dans le supérieur (PRAG).

L'idée d'une "option obligatoire» et la nécessité d'accompagner cette mesure d'une politique volontariste de recrutement ont été cruciales pour la seconde phase de la politique des langues, connue sous le nom de «politique des langues pour tous ». Ces deux principes fondateurs confirment la logique d'imposition pour amorcer la structuration de la formation en LANSAD à l'UT2J.

La logique d'imposition s'est poursuivie dans la perspective du plan quadriennal 2011-2015. Il a ainsi été décidé que tous les étudiants spécialistes d'autres disciplines devraient atteindre au minimum le niveau B2 dans une langue étrangère de leur choix, sinon à l'entrée du Master, du moins à la sortie.

\subsection{Phase 2 : massification du secteur LANSAD}

Cette politique des langues pour tous consiste ainsi à soumettre l'obtention du Master à la validation du niveau B2. Pour ce faire, la Présidence s'est dotée d'un outil administratif puissant: obliger tous les étudiants à choisir au moins une langue étrangère dans le cadre de leurs unités d'enseignements « optionnelles » et à garder cette option tant qu'ils ne pourraient justifier du niveau B $2^{11}$.

Si une augmentation des besoins pour le secteur LANSAD était prévisible à la suite de cette décision, il n'est pas certain que la Présidence en ait mesuré l'ampleur, notamment pour l'anglais.

\subsubsection{L'anglais : attracteur des masses}

Au sein de l'enseignement supérieur, l'anglais se distingue des autres langues car il subit une pression économique particulière comme le signale, par exemple, le titre d'un article du Monde daté du 2 mai $2012^{12}$ : «Un mauvais niveau d'anglais, véritable handicap pour les entreprises exportatrices». Selon une étude menée par The Economist Intelligence Unit (EIU) pour le centre de formation linguistique EF et citée dans cet article,

près d'une entreprise exportatrice sur deux a vu échouer d'importantes transactions commerciales à cause de malentendus linguistiques et culturels. [...] En France, $63 \%$ des cadres interrogés estiment que les différences de langues et de culture affectent les ambitions de leur entreprise à l'international.

L'étude mentionnée est susceptible d'être biaisée car elle a été commandée par un centre de formation linguistique. Elle illustre cependant le type de pression socio-

\footnotetext{
${ }^{11}$ Ce niveau doit être compris comme un niveau B2 «universitaire» puisque la validation du baccalauréat n'est pas suffisante : il peut être justifié soit par l'obtention d'une certification de niveau B2, soit par la validation du parcours de niveau B2 mis en place par les équipes LANSAD locales dans les différentes langues (cf. point 2 infra).

$12<$ http://www.lemonde.fr/economie/article/2012/05/02/un-mauvais-niveau-d-anglais-veritablehandicap-pour-les-entreprises-exportatrices_1694283_3234.html>
} 
économique qui pèse sur l'anglais, avec des répercussions sur l'enseignement de cette langue de l'école primaire à l'université que l'on ne retrouve pas, nous semble-t-il, pour les autres langues.

Il est donc logique que la plupart des étudiants ${ }^{13}$ choisissent l'anglais comme « option obligatoire», même dans une université offrant onze autres langues, et même s'ils l'étudient déjà depuis de très nombreuses années. Mais, in fine, c'est bien le faible niveau des étudiants en anglais, malgré un millier d'heures de cours en présentiel en collège et lycée, qui a engendré la massification de la filière LANSAD au département des études du monde anglophone (DEMA) de l'UT2J.

\subsubsection{Le faible niveau des étudiants en anglais}

Le graphique (figure 1 ci-après) présente les résultats obtenus en anglais par tous les étudiants arrivant pour la première fois à l'UT2J à la rentrée universitaire 20122013. Ces données sont issues du test « $\mathrm{ELAO}^{14}$ » que tous les étudiants doivent passer pour pouvoir s'inscrire à l'Université. Cette population de près de 3700 étudiants est composée pour moitié environ des nouveaux bacheliers et, pour autre moitié, des nouveaux étudiants s'inscrivant en L2 ou au-delà.

Les résultats présentés dans cette figure pour 2012-2013 ont été répliqués en 20132014. On observe que seuls $12 \%$ des étudiants arrivant chaque année ont au moins le niveau B2 au test ELAO. De plus, le test ELAO n'évalue pas toutes les compétences. Seules les compétences de compréhension de l'écrit et de l'oral et des connaissances lexicales et grammaticales sont évaluées. Les étudiants placés au niveau B2 par le test n'ont donc peut-être pas atteint ce niveau d'autonomie langagière dans toutes les compétences. En outre, les détails des résultats des étudiants placés au niveau B1 montrent que plus de la moitié sont au bas de l'échelle ${ }^{15}$. Ainsi près de $80 \%$ des étudiants auront besoin d'un grand nombre d'heures de travail ${ }^{16}$ pour atteindre les objectifs fixés par la Présidence.

\footnotetext{
$1375 \%$ des étudiants du LANSAD à l'UT2J choisissent l'anglais, 15\% choisissent l'espagnol, les autres langues se partagent les $10 \%$ d'étudiants restants.

${ }^{14}$ Le test ELAO, Efficient Language Assessment Online, est décrit à l'adresse suivante par l'une des entreprises qui le commercialise : <http://www.comefica.com/frcontenu/elao.htm>.

${ }^{15}$ Le test ELAO précise l'avancement de l'étudiant dans chaque niveau, par tranche de $25 \%$ (par exemple, un étudiant peut être de niveau «A2 $0 \%$ », de niveau «A2 $25 \%$ », de niveau «A2 $50 \%$ » ou de niveau «A2 $75 \%$ », après quoi il passe au niveau «B1 $0 \%$ », etc.). Le « bas de l'échelle », du niveau $\mathrm{B} 1$ que nous décrivons ci-dessus correspond à un niveau $\mathrm{B} 1$ de 0,25 ou $50 \%$.

${ }^{16}$ Selon les courbes de progression proposées par ALTE/FOREIGN OFFICE $(<\mathrm{http}: / / \mathrm{www}$.foreignoffice.fr/site/index.php?page $=$ courbe $>$ ), le passage du niveau A2 à B1 réclame en moyenne 200 heures de travail étudiant. Ce nombre croît de manière exponentielle avec l'expertise linguistique recherchée. Ces courbes sont à prendre avec précaution car le temps nécessaire de formation varie en fonction de l'individu mais elles donnent une idée globale du temps de travail requis (CECRL : 20). Une étude permettant de quantifier de manière précise les facteurs favorisant ce passage d'un niveau à un autre reste à mener.
} 
Figure 1. Répartition des étudiants LANSAD entrants à l'UT2J par niveau ${ }^{17}$ en anglais selon le test ELAO 2012-2013

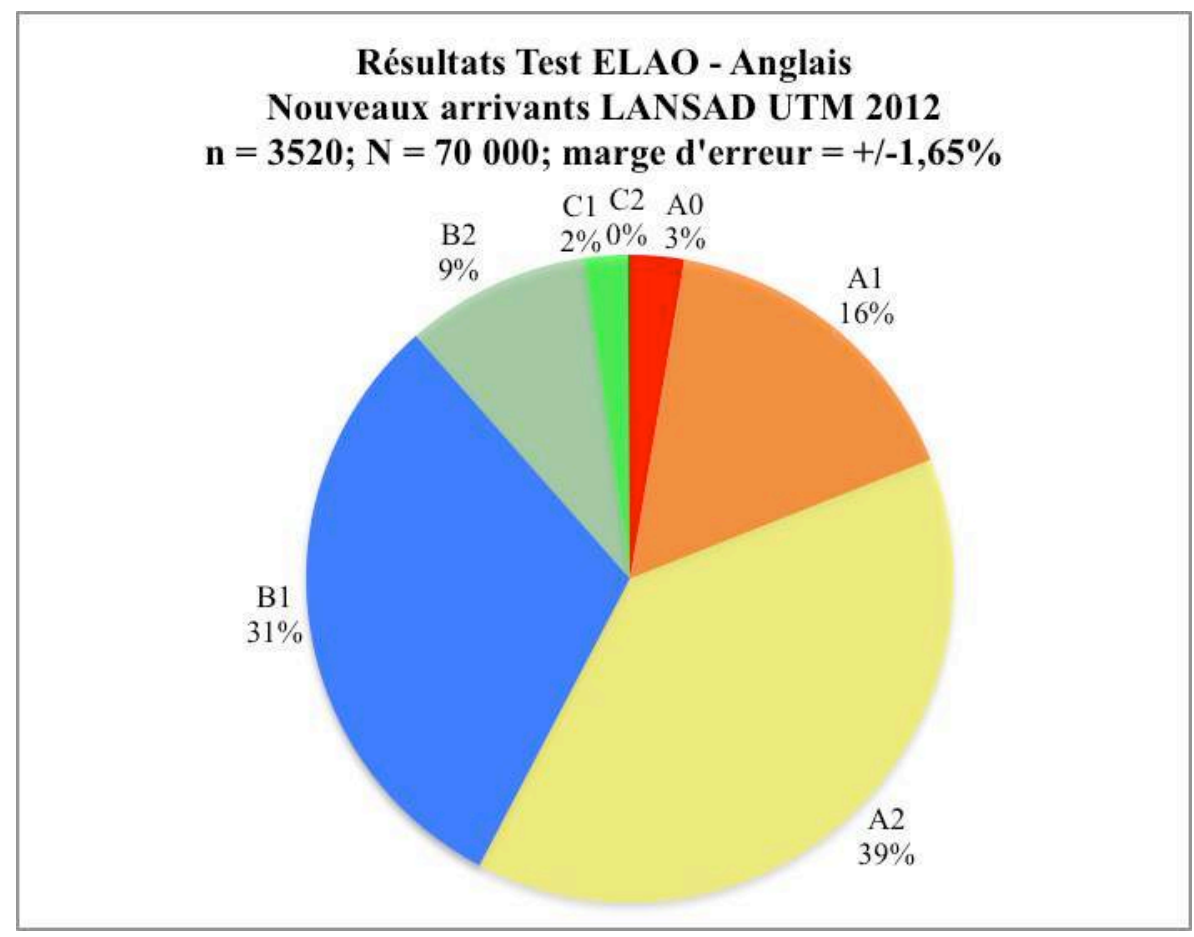

Ces chiffres ne surprendront sans doute pas les enseignants LANSAD qui font l'expérience au quotidien du faible niveau des étudiants mais ils apportent une confirmation statistique aux « impressions » de terrain. La Présidence avait-elle, de son côté, envisagé qu'en raison du faible niveau d'entrée, l'option «langue » obligatoire jusqu'au niveau B2 concernerait finalement presque la totalité des étudiants non spécialistes? Avait-elle de surcroît anticipé qu'étant donné leur niveau d'entrée, la plupart des étudiants auraient besoin de rester dans le circuit LANSAD bien au-delà d'un ou deux ans pour atteindre le niveau requis ?

\subsubsection{La massification de la formation anglais-LANSAD}

C'est donc bien la combinaison du faible niveau des étudiants à l'entrée à l'université et la décision d'imposer une option obligatoire «langue » jusqu'à la validation du niveau B2 qui a entraîné une massification ${ }^{18}$ des effectifs à l'UT2J.

\footnotetext{
${ }^{17}$ Le test ELAO propose des résultats de niveau « $\mathrm{A} 0$ » alors que ce niveau ne fait pas partie de l'échelle de niveaux du CECRL.

${ }^{18} \mathrm{Au}$ sens où cette notion est définie par le Centre national de ressources textuelles et lexicales : «Action de donner une dimension de masse à une activité réservée à une élite ; son résultat» $(<\mathrm{http}: / /$ www.cnrtl.fr/definition/massification $>$ ).
} 
Le nombre d'étudiants inscrits dans les unités d'enseignement d'anglais-LANSAD est ainsi passé de 1600 à 16000 .

Cette massification soudaine s'exprime en nombre d'individus mais aussi en volume horaire d'enseignement à assurer. Ce dernier a augmenté lui aussi considérablement pour l'anglais, passant d'environ 1800 heures ${ }^{19}$ dans la première phase de la politique des langues à environ 6000 heures dans la deuxième.

Dans une logique purement comptable, trois décisions ont pourtant été prises par la Présidence, la Direction de l'UFR et la Direction du DEMA pour endiguer cette augmentation : un nombre maximum d'étudiants dans les groupes plus élevé pour le LANSAD que pour les filières LEA et LLCE (35 au lieu de 30), une réduction drastique du nombre d'heures de cours par semestre (de $40 \mathrm{~h}$ dans la première phase à $24 \mathrm{~h}$ dans la deuxième) et un traitement «à part» des L1 par deux unités d'enseignement articulées autour du CRL (cf. point 2 infra). Sans ces trois décisions, le volume à assurer en LANSAD aurait atteint 13000 heures.

\subsection{Un paysage des langues transformé}

Malgré ces efforts pour contenir l'augmentation du volume horaire LANSAD, la politique des langues pour tous a transformé l'offre de formation en langues dans cette université qui, de par sa nature (ALLSHS), présente la particularité d'être le seul lieu de la communauté d'universités et d'établissements toulousaine où la formation LANSAD cohabite avec la formation LEA et avec la formation pour spécialistes.

\subsubsection{Nouveaux rapports de force}

Lors de la première phase de la politique des langues, la filière LANSAD anglais comptait autant d'inscrits que les filières LEA ou LLCE (respectivement 1900 et 1000 étudiants en moyenne par an), mais la «charge d'enseignement» restait limitée (1800 heures d'enseignement d'anglais en LANSAD contre respectivement environ 10000 et 12000 heures en LEA et LLCE). Une recherche privilégiant les objets scientifiques relatifs aux champs «traditionnels» de l'anglistique (civilisation, linguistique, littérature), en lien direct avec les filières LEA et LLCE, continuait donc à être légitimement favorisée.

Avec la deuxième phase de la politique des langues, les rapports de force entre les trois filières se sont trouvés bouleversés. Aujourd'hui l'anglais concerne dix fois plus d'étudiants spécialistes d'autres disciplines que d'étudiants spécialistes de la discipline : $75 \%$ des étudiants de l'UT2J qui suivent une formation en anglais sont issus d'autres UFR que l'UFR LLCE ${ }^{20}$.

\footnotetext{
${ }^{19}$ Phase $1: 1600$ étudiants sont répartis en groupes de 35 étudiants, soit 46 groupes. Chaque groupe suit $40 \mathrm{~h}$ de cours, soit un total de 1800 heures à assurer.

${ }^{20}$ Le potentiel de croissance en anglais-LANSAD est immense : le passage des groupes LANSAD à

30 étudiants comme dans les autres filières augmenterait la charge d'enseignement de 540 heures, soit
} 
Les besoins logistiques, les besoins en enseignement et les besoins en recherche ont ainsi largement évolué, créant de nouveaux rapports de nature à créer des tensions entre les filières «traditionnelles » du département d'anglais et la filière pour spécialistes d'autres disciplines. Ces tensions sont spécifiques aux universités ALLSHS puisqu'elles sont les seules à regrouper ces trois filières d'enseignement des langues. Annick Rivens Mompean (2013 : 34) évoque la « fracture » créée « de fait entre les langues pour spécialistes et les langues pour "non-spécialistes" ». Elle explique « la difficulté du secteur LANSAD à émerger dans ce contexte » (ibidem). Sur le terrain, une partie de ces tensions transparaît en particulier à travers la question des recrutements.

\subsubsection{Tensions et sous-encadrement}

La politique volontariste de recrutements en faveur de la filière LANSAD pour l'anglais, entamée en 2007, s'est poursuivie lors de la seconde phase de 2011 à 2014 avec le recrutement de deux MCF et de quatre PRAG, doublant ainsi l'équipe en place. Malgré ces recrutements, le ratio du nombre d'étudiants par rapport au nombre de titulaires révèle un sous-encadrement notoire puisque le rapport est de 1600 étudiants pour un titulaire. Le tableau 1 montre que les effectifs d'étudiants en anglais-LANSAD dans les deux autres universités de Toulouse sont comparables à ceux de 1'UT2J. Le sous-encadrement y est toutefois moins criant en raison d'un nombre de titulaires plus important.

Tableau 1. Rapport entre le nombre d'étudiants suivant une formation en anglaisLANSAD et le nombre de titulaires de cette filière à l'Université de Toulouse

\begin{tabular}{|l|l|}
\hline Université Toulouse 1 - Capitole & $\begin{array}{l}\text { Env. } 775 \text { étudiants pour } 1 \text { titulaire } \\
\text { (équipe de } 22 \text { titulaires pour env. } 17000 \text { étudiants) }\end{array}$ \\
\hline Université Toulouse 2 - Jean Jaurès & $\begin{array}{l}\text { Env. } 1600 \text { étudiants pour } 1 \text { titulaire } \\
\text { (équipe de } 10 \text { titulaires pour env. } 16000 \text { étudiants) }\end{array}$ \\
\hline $\begin{array}{l}\text { Université Toulouse 3 - Paul Sabatier } \\
\text { *FSI }\end{array}$ & $\begin{array}{l}\text { *Env. } \mathbf{3 8 5} \text { étudiants pour } 1 \text { titulaire } \\
\text { (équipe de } 39 \text { titulaires pour env. } 15000 \text { étudiants) } \\
\text { *IUT 'A' }\end{array}$ \\
$\begin{array}{l}\text { *Env. } 200 \text { étudiants pour } 1 \text { titulaire } \\
\text { (équipe de } 26 \text { titulaires pour env. 5500 étudiants) }\end{array}$ \\
\hline
\end{tabular}

Le sous-encadrement particulier du LANSAD à l'UT2J s'explique par la structuration tardive du secteur. Les recrutements pour le LANSAD à l'UT2J ont en effet commencé il y a moins d'une décennie alors qu'ils ont démarré il y a plus de 30 ans à UT1 et UT3. Les échanges lors du dernier congrès de RANACLES à Bordeaux (novembre 2013) ont révélé que la question de la structuration du LANSAD se pose aujourd'hui avec force en ALLSHS.

l'équivalent de 3 postes de MCF. Le budget du département d'anglais dépend désormais aussi de la filière LANSAD - pourvoyeur en dotation et en postes, mais relativement «économe » en charge d'enseignement. 
Au niveau national, cette structuration tardive pourrait s'expliquer par le fait que les universités ALLSHS possédaient déjà une formation en langue pour spécialistes, reléguant ainsi au second plan les quelques enseignements optionnels à dispenser aux étudiants "non-spécialistes », dont les besoins de spécialisation en langue n'étaient pas pris en compte. Ceci a des effets sur les profils de recrutement qui restent éloignés des problématiques spécifiques au LANSAD et à son corollaire, la langue de spécialité.

\subsubsection{Tensions et profils de postes}

Les postes d'enseignants-chercheurs créés pour soutenir la politique des langues pour tous à l'UT2J n'ont pas été explicitement fléchés dans un domaine de recherche en lien direct avec le LANSAD et ont finalement renforcé la recherche dans les branches traditionnelles de l'anglistique. Presque tous les MCF recrutés depuis 2007 mènent aujourd'hui leur recherche en littérature ou en civilisation, et la seule enseignante-chercheure ayant effectué des recherches en didactique de l'anglais et anglais de spécialité a été officiellement sélectionnée pour son profil de linguiste (au sens étroit du terme). Ces recrutements suggèrent qu'un lien étroit entre enseignement et recherche, pourtant fondateur à l'université, ne serait a priori pas indispensable lorsqu'il s'agit du LANSAD, pour l'institution locale en tout cas.

De plus, dans la seconde phase de la politique des langues, le rapport entre recrutement d'enseignants-chercheurs et recrutement d'enseignants du secondaire affectés dans le supérieur s'est inversé. Or l'anglais-LANSAD est le seul domaine à l'UT2J où le rapport MCF/PRAG se situe à 50/50. Cette situation ne saurait être justifiée par la masse d'heures d'enseignement à pourvoir : cette dernière est en effet si importante que la différence de temps d'enseignement entre les statuts PRAG et MCF ne permet en fait pas de combler le manque et de cesser de recourir à un très grand nombre de vacataires. Si cette tendance devait se poursuivre, elle confirmerait, nous semble-t-il, une difficulté à reconnaître la validité des recherches en didactique des langues et en langue de spécialité, domaines encore jeunes mais dont la présence est indispensable pour une structuration raisonnée de la filière LANSAD. Nous expliquons ces difficultés en ALLSHS par la présence, sur un même terrain, des formations LLCE, LEA et LANSAD et les tensions que cette cohabitation engendre.

En résumé, la politique des langues menée par la Présidence en réponse aux politiques européennes, combinée au faible niveau en langue des étudiants à l'entrée, a entraîné une massification soudaine de la filière LANSAD à UT2J et a transformé le paysage de l'enseignement des langues, notamment en anglais. Ceci n'a toutefois pas entraîné d'évolutions majeures dans le domaine de la recherche en anglistique, évolutions qui semblent pourtant indispensables pour appuyer les enseignements en LANSAD sur la recherche.

Les nouvelles conditions de travail ont imposé des contraintes dans l'élaboration de la maquette mais les nouveaux espaces de travail au CRL ont donné les moyens à 
l'équipe en place de passer d'une problématique de gestion des masses à une offre de formation individualisée.

\section{De la gestion des masses à une formation individualisée}

Selon la logique d'imposition décrite en première partie, l'équipe pédagogique a, dans un premier temps, dû concevoir dans une certaine urgence, une maquette visant à amener l'ensemble des étudiants spécialistes d'autres disciplines au niveau B2 universitaire dans une langue autre que le français. L'équipe d'anglais-LANSAD a organisé la maquette en deux pôles : l'un centré sur des enseignements en présentiel pour les étudiants de L2 à M2, l'autre articulé autour du CRL pour le public L1. L'analyse de ces deux pôles va nous permettre de faire émerger quelques-uns des mécanismes de structuration du secteur LANSAD propres au domaine ALLSHS.

\subsection{Offre de formation du L2 au M2 : gestion des masses par niveau de langue}

En raison des attentes de l'institution et de l'hétérogénéité des niveaux à l'entrée, le CECRL s'est posé comme un élément pertinent pour la structuration globale de l'offre de formation locale: il s'agissait de proposer un parcours qui pourrait permettre de valider progressivement les différents niveaux du CECRL et d'amener tous les étudiants vers le niveau B2 « universitaire », donc vers une spécialisation possible en langue, quel que soit le niveau de départ.

\subsubsection{Organisation par niveau}

Mais alors que l'équipe proposait une progression par niveau pour répondre aux besoins de chaque étudiant, et qu'elle devait faire face à un très grand nombre d'étudiants, chacun savait que les contraintes matérielles joueraient un rôle majeur dans l'organisation des enseignements : les nouveaux bâtiments de l'UFR LLCE, livrés en 2009 , ne comprendraient pas suffisamment de salles pour répondre aux besoins de toutes les langues dans toutes les filières.

Or "gérer les masses » au sein des dispositifs traditionnels que sont les cours en présentiel réclame d'avoir à sa disposition suffisamment de salles de cours. La contrainte logistique imposée par le manque de salles aurait pu susciter une réflexion sur la pertinence de développer des formations mixtes (ou «hybrides $»^{21}$ ) pour ces grands nombres d'étudiants, pourquoi pas avec l'appui de $\mathrm{MOOC}^{22}$. Toutefois,

${ }^{21}$ Voir par exemple les travaux menés à Nantes au sein du CRINI par Sophie Belan et MarieFrancoise Narcy-Combes (2011), Jemma Buck et Julie McAllister (2012), Rebecca Starkey-Perret, Julie McAllister et Marie-Françoise Narcy-Combes (2012) ou par Elke Nissen (2012).

${ }^{22}$ Comme les plateformes pédagogiques de type Moodle, les MOOC permettent de communiquer un certain nombre d'informations, de savoirs et de savoir-faire et d'interagir à distance sur un mode synchrone ou asynchrone. On rompt ainsi avec l'utilisation traditionnelle des espaces physiques, par exemple, en dispensant un cours sur la base de regroupements non pas hebdomadaires mais mensuels ou bi-mensuels. On peut toutefois interroger la plus-value «technique » et la différence réelle par rapport à un cours proposé sur une plateforme pédagogique telle que Moodle et qui serait ouvert à un 
l'urgence dans laquelle l'équipe LANSAD s'est vue devoir travailler au moment de la conception de la maquette n'était pas de nature à favoriser une telle réflexion.

Celle-ci s'est donc opérée sur un mode plus «gestionnaire»: il s'agissait de prévoir une maquette qui permettrait, au moins dans un premier temps, de " gérer les masses » d'étudiants d'anglais-LANSAD dans un nombre de salles de cours restreint. Or, plus l'on imposerait de critères sur la formation des groupes, plus il serait difficile d'en optimiser la gestion. En construisant la maquette autour des niveaux du CECRL, on imposait déjà un critère important. Toute contrainte supplémentaire aurait pesé lourdement sur l'organisation. C'est ainsi que l'équipe a décidé de mélanger, au sein d'unités d'enseignements par niveaux du CECRL, les étudiants de toutes les années (du L2 au M2) et de toutes les disciplines (psychologie, sociologie, histoire, arts, philosophie, etc.).

\subsubsection{Objet d'étude}

Le texte approuvé par la Société des anglicistes de l'enseignement supérieur sur l'évolution et les enjeux des formations et de la recherche dans le secteur LANSAD rappelle que le terme «secteur LANSAD » peut revêtir "des réalités différentes selon les institutions» (2011: 2). Il peut correspondre à "un enseignement à des étudiants d'une même discipline » (ibidem). Dans ce cas :

l'articulation entre ces formations dans un domaine clairement identifié et un type particulier de discours spécialisé s'avère source d'enrichissement à la fois pour la discipline visée $[\ldots]$ et pour le développement des compétences linguistiques et langagières [...]. (ibid)

L'objectif de maîtrise de l'anglais de spécialité est alors reconnu comme constitutif du secteur LANSAD et la finalité des enseignements « est de permettre l'insertion linguistique et culturelle $\mathrm{du}$ futur professionnel qu'est l'étudiant dans l'environnement socio-professionnel de sa spécialisation » (ibid).

Le second cas mentionné par le texte est celui d'un « enseignement dédié à des étudiants issus de disciplines variées " (ibid). Dans ce cas, qui correspond à celui de l'UT2J, «l'ancrage disciplinaire est plus flou, voire absent» (ibid). Cette situation est précisée par Michel Van der Yeught (2014 : §27) :

[...] dans de nombreux cas, l'adéquation LANSAD/LSP est totalement inexistante.
Certains cursus adossent une langue à des formations disciplinaires sans que l'on y
enseigne la langue de spécialité correspondante. D'autres dispensent des cours de
langues à des étudiants de disciplines différentes réunis dans les mêmes groupes. Dans
ces situations, très fréquentes en sciences humaines, la notion de LSP disparaît.

En réduisant les contraintes sur la constitution des groupes LANSAD à l'UT2J, on optimisait la gestion logistique des salles mais on évacuait dans le même temps, de

très grand nombre d'étudiants et, finalement, ouvert à tous ceux qui souhaitent y participer. Deux liens pour alimenter le débat: <https://moodle.org/mod/forum/discuss.php?d=204799> et $<$ http://fr.slideshare.net/iaindoherty/moodle-in-a-massively-open-world $>$. 
facto, la question de l'anglais de spécialité. Toutefois, l'absence d'anglais de spécialité s'explique également par une décision collective des enseignantschercheurs en poste de lier leur domaine de recherche à leurs enseignements en LANSAD. Les politiques de recrutement locales ayant engendré le recrutement d'enseignants-chercheurs n'effectuant pas leur recherche en anglais de spécialité en lien avec le LANSAD, cet objet d'enseignement n'a pas trouvé sa place dans la maquette. L'absence de la langue de spécialité des étudiants dans la maquette actuelle est donc avant tout le résultat de la culture de recherche des universités ALLSHS, et des recrutements qu'elle a engendrés (cf. supra, 1.3.3).

\subsubsection{Contenus}

Les contenus des enseignements d'anglais par niveau pour les étudiants de L2 à M2 ont été dès lors définis en lien avec les domaines de recherche des MCF en poste, autour de thématiques de recherche issues des branches traditionnelles de l'anglistique (littérature et civilisation) ou transversales (études filmiques, études sur le genre).

L'unité d'enseignement pour valider la première partie d'un niveau B1 « universitaire» (compétences écrites), "Daily lives unsettled by extraordinary events », s'appuie par exemple sur des textes littéraires et civilisationnels en rapport avec le thème annoncé : la vie quotidienne pendant la Seconde guerre mondiale en Grande Bretagne, le trauma du 11 septembre ou « la grande famine » en Irlande. L'unité d'enseignement pour valider la seconde partie du niveau B1 universitaire (compétences orales), «Artists' reactions to our world», s'inspire de travaux de recherche intersémiotique sur la circulation entre les arts (littérature, arts visuels, musique). L'unité d'enseignement « Minorities » (niveau B2 universitaire, compétences orales) est fondée sur les questionnements actuels de la recherche en civilisation américaine sur les concepts de genre, de race et de classe.

Toutes les unités d'enseignement de «niveau», dont les contenus sont intrinsèquement liés aux profils des enseignants-chercheurs recrutés, offrent aujourd'hui aux étudiants du L2 au M2 une progression raisonnée vers le niveau universitaire B2. Seules les unités d'enseignement "ANPOS1LX» et «ANPOS2LX» n'entrent pas dans ce système de «niveau». Ces unités d'enseignement d'anglais ( $\mathrm{AN} »)$ dites de positionnement» («PO ») pour le premier semestre (« $\mathrm{S} 1)$ et le deuxième semestre (« $\mathrm{S} 2 »)$ s'adressent exclusivement aux étudiants d'anglais-LANSAD de première année. Elles comptaient chacune 2500 étudiants en 2013-2014 et elles présentent la particularité de proposer à ces étudiants une formation individualisée sur le modèle des formations en centres de langues.

\subsection{Offre de formation pour les $\mathrm{L} 1$ : une formation individualisée de masse}

Les universités restent aujourd'hui les seules institutions de l'enseignement supérieur qui ne pratiquent pas une forme de sélection à l'entrée autre que le baccalauréat. Selon les indicateurs du MESR (2013: 33), le public L1 y est 
extrêmement hétérogène : $80 \%$ provient de baccalauréats généraux, $13 \%$ de baccalauréats technologiques et $7 \%$ de baccalauréats professionnels ${ }^{23}$.

\subsubsection{Prémisses d'une unité d'enseignement d'autoformation guidée}

Dans les universités ALLSHS, ces effectifs L1 sont souvent non seulement pléthoriques mais aussi «instables» puisque le taux d'abandon y est plus élevé qu'ailleurs (idem : 48). La problématique de gestion de ces étudiants particuliers a encouragé l'équipe pédagogique à organiser une unité d'enseignement spécifique aux L1 sur le mode de l'autoformation guidée au CRL.

La construction de cette offre de formation individualisée s'est appuyée sur les travaux menés autour des CRL, notamment dans l'objectif de développer chez l'étudiant la «capacité à apprendre » en favorisant un processus d'autonomisation pour et par l'apprentissage de l'anglais en autoformation guidée :

$[\ldots][E]$ st « autonome » un apprenant qui sait apprendre. Les pratiques mises en place dans cette orientation ont pour visée l'autonomisation de l'apprenant, c'est-à-dire le développement de sa capacité d'apprendre. (Holec 1990 : 77)

Deux outils clés pour guider le travail individuel articulé autour du CRL ont permis de structurer l'unité d'enseignement: le carnet de bord (Macré 2009; Rivens Mompean \& Eisenbeis 2009 ; Chateau \& Zumbihl 2010), appelé «logbook» mais envisagé comme un outil de réflexion sur l'apprentissage, et l'entretien de conseil (Gremmo 2003 et 2009; Ciekanski 2005), visant lui aussi à accompagner les étudiants dans leur travail en langue. Des ateliers de conversation en petits groupes avec des locuteurs natifs ont également été proposés, en plus des ressources disponibles au CRL et sur l'espace-cours de la plateforme Moodle.

Malgré la mise à disposition d'outils typiques d'une autoformation guidée, les deux premières années d'expérimentation de cette unité d'enseignement se sont soldées par un sentiment d'échec chez les étudiants comme chez les enseignants. Les évaluations de fin de semestre, au moyen d'un entretien oral de cinq minutes ${ }^{24}$ sur la base du carnet de bord, ont en effet montré que la plupart des étudiants n'avaient pas engagé un processus d'autonomisation, notamment en raison d'une incompréhension des objectifs et des sentiments de désordre et d'abandon ressentis durant toute la première moitié du semestre.

Une réappropriation de cette offre d'autoformation guidée s'est amorcée, notamment en prenant en compte le paradoxe de demander aux étudiants a priori les moins autonomes (les L1) de s'engager dans un processus d'autonomisation alors même qu'ils découvrent un environnement et un univers totalement nouveaux.

${ }^{23}$ Ceci représente en fait $50 \%$ des bacheliers de filières professionnelles qui entrent dans l'enseignement supérieur.

${ }^{24}$ Entretiens nécessairement courts en raison du nombre d'étudiants (2500 créneaux de 5 minutes doivent être prévus) mais qui permettent toutefois d'évaluer si l'étudiant/e est engagée dans un processus d'autonomisation et de discuter des grandes lignes du dispositif proposé. 


\subsubsection{Innovation pour une autoformation individualisée de masse}

L'analyse du parcours possible d'un étudiant d'anglais-LANSAD lambda qui souhaiterait, par exemple, améliorer sa compréhension de l'anglais oral (Terrier 2011) a montré que les outils «typiques » de l'autoformation, tels qu'ils avaient été appliqués à l'UE, étaient insuffisants pour permettre à cet étudiant lambda d'atteindre son objectif. Trois problèmes structurels ont été identifiés à travers l'exemple de la compétence de compréhension de l'anglais oral :

- l'inadéquation entre l'objectif de "gestion des masses », qui faisait de cette unité d'enseignement une "gare de triage" pour faire face aux effectifs instables d'étudiants au premier semestre du L1, et la volonté de l'équipe pédagogique de proposer une offre de formation raisonnée articulée autour du CRL ;

- le manque d'outils de formation (et d'information) adaptés aux étudiants à qui s'adressait le dispositif et qui n'a pas permis de respecter les «sept piliers de l'autoformation» tels qu'ils ont été définis par Philippe Carré et Michael Pearn (1992, cités dans Rivens Mompean 2013 : 141), notamment par une présentation

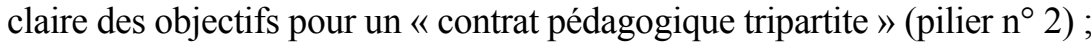

- le paradoxe de proposer aux étudiants les moins autonomes (étudiants arrivant à l'université) une unité d'enseignement dans laquelle l'autonomie est non seulement une fin mais aussi un moyen (Barbot $2001: 22$ ).

Ces trois problèmes semblaient tous être liés à une même cause : la difficulté intrinsèque de proposer une formation individualisée à des masses d'étudiants. La question qui s'est posée à l'équipe a donc été celle de l'adaptation d'un dispositif de formation individualisée pour une masse d'étudiants de L1. La réflexion menée a soulevé la question d'un encadrement plus fort des étudiants afin de s'assurer que tous bénéficieraient d'un socle minimal d'informations sur l'unité d'enseignement et de formation méthodologique pour se saisir du dispositif d'autoformation guidée proposé.

C'est ainsi qu'a émergé l'idée d'utiliser un outil traditionnel de gestion des masses à l'université : les cours magistraux en amphithéâtre à la suite des travaux de Linda Terrier (2011: 549) qui soulevaient la question «de la répartition des activités d'enseignement/apprentissage de la compréhension de l'oral au sein des différents 'espaces' et 'temps' de langues aujourd'hui disponibles dans les universités ». Parmi ces différents espaces-temps disponibles dans les universités se trouvent les cours magistraux en amphithéâtre. La réflexion a donc abouti à la mise en place de la seconde version de l'unité d'enseignement d'autoformation guidée dont la particularité est de mêler les outils traditionnels de gestion des masses (tels que les amphithéâtres) aux outils désormais traditionnels d'individualisation des parcours (TIC, entretien individuel, carnet de bord, etc.).

Cet agencement inédit d'outils existants pour résoudre les problèmes rencontrés durant les deux premières années de l'unité d'enseignement correspond au 
processus d'innovation tel que défini par Rivens-Mompean (2013) à la suite des travaux de Brigitte Albero. Le changement opéré ici est innovant dans la mesure où il « permet de résoudre un problème ou de répondre à une situation pour laquelle les moyens en place antérieurement ne suffisent pas » (idem: 88). L'auteure (id. : 87) rappelle qu'innovation n'est pas nécessairement synonyme de "nouveauté » mais, qu'en revanche, "l'agencement inédit d'objets et de pratiques parfois connus dans d'autres arrangements ou autres contextes » peut être source d'innovation. C'est exactement dans ce cas de figure que nous nous situons.

\subsubsection{Les piliers de l'autoformation}

En plus des trois problèmes structurels liés à la masse d'étudiants impliqués dans le dispositif d'autoformation guidée, l'équipe a également fait le constat qu'il manquait au dispositif un espace essentiel d'apprentissage méthodologique pour favoriser l'autonomisation des étudiants: des séances pour "apprendre à apprendre » - le troisième pôle incontournable de l'apprentissage au CRL selon Annick Rivens Mompean (2012 : 72).

Des cours magistraux obligatoires pour tous et répartis régulièrement tout au long $\mathrm{du}$ semestre sont alors apparus comme le moyen d'offrir une première aide méthodologique indispensable à l'ensemble des étudiants de L1 pour favoriser leur autonomisation. Un renforcement de ces CM par des ateliers de méthodologie au CRL (optionnels mais fortement recommandés) et des modules de travail sur Moodle ont également été prévus. Le cours magistral, outil traditionnel permettant de "gérer les masses", a ainsi été transformé en un espace pour apprendre à apprendre.

Contrairement à la première version de l'unité d'enseignement d'autoformation guidée, l'UE ANPOS1LX répond aujourd'hui aux sept piliers de l'autoformation tels que modélisés par Philippe Carré et Michael Pearn (1992, cités dans Rivens Mompean 2013 : 141) et décrits ci-dessous.

1. Le projet individuel est pris en compte à travers l'entretien individuel de conseil obligatoire pour tous les étudiants.

2. Le contrat pédagogique est clairement exposé lors du premier cours magistral et rediscuté lors de l'entretien.

3. Le premier cours magistral génère un mécanisme de préformation en sensibilisant les étudiants au dispositif d'autoformation guidée proposé.

4. L'UE comprend des formateurs-facilitateurs, enseignants et personnels du CRL qui ont, pour la première fois à l'UT2J, reçu à la rentrée 2013 une formation en lien explicite avec les unités d'enseignement de formation LANSAD.

5. Un cours "dédié » sur la plateforme Moodle, des logiciels acquis sous l'impulsion des responsables de l'unité d'enseignement ANPOS1LX et les TIC au service de l'enseignement/apprentissage d'une langue permettent d'offrir un 


\section{environnement ouvert de formation à distance.}

6. L'alternance individu/collectif est assurée par les quatre regroupements prévus en cours magistraux ${ }^{25}$ dans le semestre.

7. Enfin, un triple niveau de suivi (sujet, groupe et institution) est assuré pour favoriser le respect du contrat pédagogique tripartite.

Le contrat d'autoformation guidée semble ainsi rempli par l'institution malgré des effectifs pléthoriques. Afin de le renforcer, le travail en autoformation a été étalé sur l'ensemble de l'année, donnant ainsi le temps aux étudiants de se familiariser avec les outils à leur disposition et d'apprendre leur métier d'étudiant. L'UE du second semestre (ANPOS2LX) intègre d'ailleurs au processus d'autonomisation en langue les problématiques universitaires d'insertion professionnelle et de langue de spécialité à travers un travail individuel par projets.

C'est donc par l'innovation que les problèmes identifiés dans la première version de l'unité d'enseignement d'autoformation guidée ont pu être sinon résolus ${ }^{26}$, du moins abordés de manière raisonnée. La prise en compte des spécificités institutionnelles locales a ainsi permis à l'équipe de passer d'une logique d'imposition à une logique de (ré)appropriation de l'offre pour proposer une formation raisonnée des masses de L1. Ces contraintes locales ont également pesé fortement sur l'organisation de la formation des L2 aux M2. Elles apparaissent comme fondamentales dans les mécanismes de structuration du secteur LANSAD en ALLSHS.

\subsection{Structuration de l'offre de formation dans le cadre didactique contraint du LANSAD en ALLSHS}

L'analyse des enjeux propres à 1'UT2J et du dispositif d'autoformation mis en place pour les L1 nous permet de proposer des éléments d'analyse de la structuration du secteur LANSAD potentiellement généralisables à l'ensemble des universités ALLSHS.

\subsubsection{Le cadre didactique contraint du LANSAD enrichi}

Lors du congrès du GERAS 2013, Linda Terrier a montré que l'analyse du contexte institutionnel large des formations LANSAD permettait de décrire le « cadre didactique contraint du LANSAD » selon quatre critères (voir tableau 2).

\footnotetext{
${ }^{25}$ Les cours magistraux 2, 3 et 4 sont consacrés aux problématiques méthodologiques autour de la compréhension de l'oral, de l'acquisition du lexique et de la phonologie de l'anglais.

${ }^{26}$ Le nouveau dispositif d'autoformation guidée sur l'année a été mis en place à la rentrée 2013. Des outils d'évaluation sont prévus. Ce travail s'inscrit dans une démarche de recherche-développement qui concerne à la fois «la formation de modèles théoriques pour parvenir à des préconisations didactiques et l'évaluation de l'offre technico-pédagogique pour l'apprentissage des langues» (Guichon 2007 : 38).
} 
Tableau 2. Cadre didactique contraint du LANSAD

\begin{tabular}{|l|l|}
\hline Critères & Caractéristiques propres au secteur LANSAD (pour l'anglais) \\
\hline $\begin{array}{l}\text { Heures de cours en } \\
\text { présentiel }\end{array}$ & $\begin{array}{l}\text { 20 à 30 heures / semestre } \\
\text { (semestres de 12 à 16 semaines) }\end{array}$ \\
\hline Public & $\begin{array}{l}\text { L1 à M2 } \\
\text { Spécialistes d'autres disciplines que l'anglais } \\
\text { Niveau en anglais hétérogène } \\
\text { Motivation hétérogène après plusieurs années d'anglais }\end{array}$ \\
\hline Ressources & $\begin{array}{l}\text { Cours en présentiel (CM, TD, TP dans salles « traditionnelles » } \\
\text { et/ou laboratoires multimédias de langue) } \\
\text { Plateformes pédagogiques } \\
\text { Centre de langues } \\
\text { Enseignement / apprentissage mixte (blended learning) }\end{array}$ \\
\hline Objet d'étude & \begin{tabular}{l} 
Anglais de spécialité \\
\hline
\end{tabular}
\end{tabular}

Cadre proposé lors d'une communication au congrès du GERAS (Terrier 2013).

L'analyse de la politique des langues telle qu'elle a été mise en place à l'UT2J ne réclame pas, selon nous, de modifier ces critères. Par exemple, même si, en raison des profils des enseignants-chercheurs recrutés, la maquette locale n'intègre pas la notion d'anglais de spécialité, l'anglais de spécialité comme objet d'étude peut difficilement être remis en cause, du moins comme objectif à moyen ou long terme (voir texte commission de formation SAES mentionné supra).

L'analyse du processus de massification de l'anglais-LANSAD à l'UT2J et de l'offre de formation qui en a découlé nous semble en revanche de nature à enrichir ce «cadre didactique contraint du LANSAD ». Certains éléments semblent en effet généralisables à l'ensemble du secteur LANSAD. Ils sont notés en gras dans le tableau 3.

Tableau 3. Cadre didactique contraint du LANSAD enrichi

\begin{tabular}{|l|l|}
\hline Critères & $\begin{array}{l}\text { Caractéristiques propres au secteur LANSAD (pour } \\
\text { l'anglais) }\end{array}$ \\
\hline Attentes institutionnelles & $\begin{array}{l}\text { Maîtrise de l'anglais pour la mobilité au sein de l'EEES, } \\
\text { l'employabilité et l'efficacité socio-économique }\end{array}$ \\
\hline Objectif de la formation & Atteindre un niveau B2 « universitaire » \\
\hline $\begin{array}{l}\text { Heures de cours en } \\
\text { présentiel }\end{array}$ & $\begin{array}{l}20 \text { à } 30 \text { heures / semestre } \\
\text { (semestres de } 12 \text { à } 16 \text { semaines) }\end{array}$ \\
\hline Forces en présence & $\begin{array}{l}\text { Équipes LANSAD réduites } \\
\text { chronique) } \\
\text { «Secondarisation }{ }^{27} \text { des équipes }\end{array}$ \\
\hline
\end{tabular}

${ }^{27}$ Le terme «secondarisation» exprime le poids plus fort des PRAG et PRCE dans les filières 


\begin{tabular}{|c|c|}
\hline Public & $\begin{array}{l}\text { L1 à M2 } \\
\text { Spécialistes d'autres disciplines que l'anglais } \\
\text { Niveau en anglais hétérogène } \\
\text { Niveau d'entrée plutôt faible (80\% d'étudiants au } \\
\text { " niveau seuil » voire en deçà) } \\
\text { Motivation hétérogène après plusieurs années d'anglais }\end{array}$ \\
\hline Ressources & $\begin{array}{l}\text { Cours en présentiel (CM, TD, TP dans salles } \\
\text { «traditionnelles » et/ou laboratoires multimédias de langue) } \\
\text { Plateformes pédagogiques } \\
\text { Centre de langues } \\
\text { Enseignement / apprentissage mixte (blended learning) }\end{array}$ \\
\hline Objet d'étude & Anglais de spécialité \\
\hline
\end{tabular}

2.3.2. Un cadre didactique contraint spécifique pour le LANSAD en ALLSHS ?

Le tableau 3 se veut être un essai de définition d'un cadre institutionnel "large » commun à un grand nombre de formations LANSAD au niveau national. Certains éléments de la situation locale ne peuvent pas y être inscrits car ils ne reflètent pas nécessairement la situation du secteur LANSAD dans la majorité des établissements de l'enseignement supérieur français. Ils pourraient toutefois se révéler typiques d'un cadre didactique contraint du LANSAD dans les universités ALLSHS :

- la massification soudaine ${ }^{28}$ à laquelle les personnels enseignants et administratifs ont dû faire face sur le terrain ;

- le sous-encadrement en LANSAD plus fort en ALLSHS que dans les autres domaines du LANSAD, toutes choses étant égales par ailleurs (voir tableau 1);

- une politique de recrutement qui ne favorise pas l'adéquation entre profil de recherche et profil d'enseignement pour le LANSAD.

Nous avons montré que ces trois éléments étaient tous liés à la cohabitation de la filière LANSAD avec les filières pour spécialistes (LEA et LLCE), qui constitue l'une des spécificités des universités ALLSHS. Ils contribueraient alors à la définition d'un «cadre didactique contraint du LANSAD-ALLSHS » qui serait, sinon en partie distinct du cadre didactique du LANSAD pour d'autres domaines de spécialité, du moins marqué plus fortement par ces éléments là.

Cette hypothèse mérite, pensons-nous, d'être explorée plus avant dans de prochains travaux de recherche car nous partageons avec M. Van der Yeught (2014 : §27) l'idée que «le LANSAD reste un territoire pédagogique immense aux contours imprécis et aux caractéristiques extrêmement hétérogènes ».

LANSAD par rapport aux autres filières.

${ }^{28}$ Cette massification a également eu lieu dans les UFR autres que ALLSHS dès 2002, mais ses effets ont été moins visibles en raison des formations déjà en place avant la réforme LMD. 
Il conviendrait alors de continuer à identifier les «facteurs unificateurs » (ibidem) du secteur LANSAD, mais aussi les éléments qui, au-delà de situations locales, permettraient de créer des "sous-ensembles», au sens culiolien du terme, en fonction des différents (grands) domaines de spécialisation du LANSAD.

\section{Conclusion}

En même temps que la Présidence de 1'UT2J décidait de mettre en place la politique des langues pour tous, elle inaugurait la nouvelle UFR LLCE, comprenant le Centre de Ressources des Langues. Un nouvel outil de travail se dessinait donc au moment où l'équipe LANSAD devait construire une maquette cohérente en lien avec l'objectif de niveau B2 pour tous les étudiants. Comme nous l'avons vu, ces nouvelles conditions matérielles de travail ont largement influencé l'offre de formation du L2 au M2 et la prise en charge des étudiants de L1 par un dispositif d'autoformation guidée et encadrée.

La mise à disposition de nouveaux outils de travail ne saurait toutefois suffire à expliquer la structuration d'une offre de formation. Nous avons montré que c'est bien la massification du secteur LANSAD et la présence de plus de 2500 étudiants dans une même unité d'enseignement qui ont encouragé les enseignants à travailler différemment. Et, alors que le recours au CRL apparaissait au départ comme la réponse logistique pour gérer les masses, l'intégration des résultats de la recherche en didactique des langues a permis de proposer une structuration innovante de la formation. C'est ainsi qu'une décision politique éminemment descendante et des contraintes structurelles fortes ont, finalement, engendré des innovations pédagogiques en lien avec la recherche. Aujourd'hui, ces innovations sont, à leur tour, peu à peu intégrées à d'autres unités d'enseignement et créent une dynamique dans toute l'UFR, dans le mouvement ascendant décrit par Guichon (2012).

L'analyse des tensions politiques, structurelles, didactiques et pédagogiques a finalement permis d'établir le "cadre didactique contraint du LANSAD enrichi » dans lequel l'offre de formation LANSAD à l'UT2J a été développée. Nous pensons que ce cadre enrichi est généralisable au niveau national et qu'il peut alimenter la réflexion sur la structuration des formations LANSAD.

Cette structuration dépendrait donc à la fois du cadre didactique contraint du LANSAD et des spécificités locales, institutionnelles comme matérielles, mais aussi des avancées de la recherche en didactique des langues LANSAD. La place de cette recherche aux côtés des recherches fondamentales et appliquées menées dans les filières LLCE et LEA et sa reconnaissance par l'ensemble des acteurs de l'enseignement supérieur restent ainsi l'un des enjeux majeurs pour l'avenir. 


\section{Références}

Bailly, D. 1998. Les mots de la didactique des langues. Le cas de l'anglais, Lexique. Paris : Ophrys.

Barbot, M.-J. 2001. Les auto-apprentissages. CLE International, Paris.

Belan, S. \& M.-F. Narcy-Combes. 2011. «Accompagnement et innovation : quels outils pour un suivi efficace dans un dispositif hybride? ». Mélanges CRAPEL $n^{\circ} 32: 85-99$.

Buck, J. \& J. Mc Allister. 2012. «Mise en place d'un dispositif d'apprentissage hybride à l'université ». Cahiers de l'APLIUT, Vol. XXX N $1 \mid$ 2011 : 83-101.

Chateau, A. \& H. Zumbihl. 2010. «Le carnet de bord, un outil permettant le cheminement vers l'autonomisation dans un dispositif d'apprentissage de l'anglais en ligne ?» Alsic [En ligne], Vol. $13 \mid 2010$, mis en ligne le 28 février 2010. URL : <http://alsic.revues.org/1392> ; DOI : 10.4000/alsic.1392

Ciekanski, M. 2005. L'accompagnement à l'autoformation en langue étrangère : contribution à l'analyse des pratiques professionnelles. Doctorat de Sciences du Langage. Nancy : Université Nancy 2.

Conseil de l'Europe. 2001. Cadre européen commun de référence pour les langues : Apprendre, Enseigner, Evaluer. Paris : Didier.

Gremmo, M.-J. 2003. « Aider l'apprenant à mieux apprendre : le rôle du conseiller ou le discours comme lieu de rencontre pédagogique». In Albero, B. (coord.). Autoformation et enseignement supérieur. Paris, Hermès, 153-166.

Gremmo, M.-J. 2009. «Conseiller en langues: proposition d'analyse de deux décennies de théorie et de pratique(s) pour une approche comparée du tutorat en FOAD ». In Rivens Mompean, A. \& Barbot, M.-J. (dirs.) Dispositifs médiatisés en langues et évolutions professionnelles pour l'accompagnement-tutorat. Villeneuve d'Asq : Université Lille 3, 173-190.

Guichon, N. 2007. «Recherche-développement et didactique des langues». Les Cahiers de l'Acedle, $n^{\circ} 4: 37-54$.

Guichon, N. 2012. «Les usages des TIC par les lycéens - déconnexion entre usages personnels et usages scolaires ». Revue STICEF, Volume 19, 2012, ISSN : 1764-7223, mis en ligne le 20/10/2012, <http://sticef.org>

Holec, H. 1990. «Qu'est-ce qu'apprendre à apprendre?». Mélanges CRAPEL, $n^{\circ} 20: 75-87$.

Macré, N. 2009. «L'analyse d'un dispositif de langues à travers des "carnets de bord" des apprenants de niveau A2 ». Les Cahiers de l'Acedle, vol. 6, $n^{\circ} 1$ : 131-152.

MESR. 2013. L'état de l'enseignement supérieur et de la recherche en France, $n^{\circ} 6$, février 2013

$<\mathrm{http}: / /$ multimedia.enseignementsuprecherche.gouv.fr/evaluation_statistiques/etat_enseignement_suprecherche/index.html $>$ (consulté le 15 jan. 2013). 
Nissen, E. 2012. «Autonomie dans une formation hybride: qu'en dit l'apprenant? ». Les langues modernes $n^{\circ} 3 / 2012: 18-27$.

Rivens Mompean, A. 2012. "Le CRL : un espace privilégié pour aller vers l'autonomie ». Les langues modernes $n^{\circ}$ 3/2012: 69-74.

Rivens Mompean, A. 2013. Le Centre de Ressources en Langues: vers la modélisation $d u$ dispositif d'apprentissage. Villeneuve d'Ascq: Presses Universitaires du Septentrion.

Rivens Mompean, A. \& M. Eisenbeis. 2009. "Autoformation en langues : quel guidage pour l'autonomisation? ». Les cahiers de l'ACEDLE, vol. 6, $n^{\circ} 1: 221$ 244.

Société des Anglicistes de l'Enseignement Supérieur. 2011. Évolution et enjeux des formations et de la recherche dans le secteur LANSAD. $<\mathrm{http} / / /$ sha.univpoitiers.fr/saesfrance/spip.php?article262> (consulté le 15 jan. 2013).

Starkey-Perret, R., J. McAllister \& M.-F. Narcy-Combes. 2012. «Représentations des enseignants d'anglais et évaluation d'un dispositif hybride : image de soi, image de l'apprenant et appropriation du dispositif». Recherche et pratiques pédagogiques en langues de spécialité, Volume XXXI N 1 | 2012:74-96.

Taillefer, G. 2009. «Le CRL et la démarche qualité : perspective européenne ». In Rivens, A. \& M.-J. Barbot (dir.). Dispositifs médiatisés en langues et évolutions professionnelles pour l'accompagnement-tutorat. Villeneuve d'Asq : Université Lille 3, 69-90.

Terrier, L. 2011. « Méthodologie linguistique pour l'évaluation des restitutions et analyse expérimentale des processus de didactisation du son. Recommandations pour un apprentissage raisonné de la compréhension de l'anglais oral par les étudiants francophones du secteur LANSAD ». Thèse de Doctorat de $3^{\mathrm{e}}$ cycle. Toulouse : Université Paul Sabatier Toulouse 3.

Terrier, L. 2013. «La compréhension de l'anglais oral ou l'abolition de frontières ». Communication au $34^{\mathrm{e}}$ Colloque du GERAS, «Domaines, territoires et frontières ». 22 mars 2012, ENS Cachan.

Van der Yeught, M. 2014. "Développer les langues de spécialité dans le secteur LANSAD - Scénarios possibles et parcours recommandé pour contribuer à la professionnalisation des formations ». Recherche et pratiques pédagogiques en langues de spécialité, Vol. XXXIII $N^{\circ} 1 \mid 2014$. [En ligne], mis en ligne 15 mars 2014. URL : <http://apliut.revues.org/4153> (consulté le 1 nov. 2014).

\section{Annexe 1 : glossaire des acronymes}

ALLSHS : arts, lettres, langues, sciences humaines et sociales

CLES : certificat de compétences en langue de l'enseignement supérieur

$\mathrm{CM}$ : cours magistral

CRL : centre de ressources en langues et «Centre de Ressources des Langues» (nom officiel du CRL de l'UT2J)

DEMA : département des études du monde anglophone de l'UT2J 
ELAO : Efficient Language Assessment Online

GERAS : groupe d'étude et de recherche en anglais de spécialité

IUT : institut universitaire de technologie

L1 : première année de licence

L2 : deuxième année de licence

LANSAD : langue pour spécialistes d'autres disciplines

LEA : langues étrangères appliquées

LLCE : langue, littérature et civilisation étrangères

LMD : licence - master - doctorat

LSP : langue de spécialité

M2 : deuxième année de master

MCF : maître de conférences, enseignant-chercheur à l'université

MESR : ministère de l'enseignement supérieur et de la recherche

MOOC : Massive Open Online Course

PRAG : professeur agrégé affecté dans l'enseignement supérieur

RANACLES : rassemblement national des centres de langues de l'enseignement supérieur

SAES : société des anglicistes de l'enseignement supérieur

TD : travaux dirigés

TIC : technologies de l'information et de la communication

TP : travaux pratiques

UE : unité d'enseignement

UFR : unité de formation et de recherche

UT1 : université Toulouse 1 - Capitole (droit, économie, gestion)

UT2J : université Toulouse 2 - Jean Jaurès

UT3 : université Toulouse 3 - Paul Sabatier (sciences)

Linda Terrier est Maître de conférences à l'Université Toulouse - Jean Jaurès. Elle assure son enseignement en LANSAD et en linguistique en LLCE du L1 au M1. Elle est responsable des UE ANPOS1LX et ANPOS2LX d'auto-formation guidée pour les étudiants de L1 en anglaisLANSAD. Elle effectue sa recherche en linguistique et en didactique de l'anglais au sein du laboratoire cultures anglo-saxonnes (CAS EA 801). Ses travaux sur la compréhension de l'anglais oral par les étudiants francophones l'ont amenée à se concentrer sur la question de l'intégration des TIC. Elle travaille aujourd'hui à transférer ses travaux en didactique pour le LANSAD et son expérience de cet enseignement aux problématiques de l'enseignement de la linguistique de l'anglais pour les spécialistes de la discipline.

$<$ linda.terrier@univ-tlse2.fr $>$

Cristelle Maury est Maître de conférences à l'Université Toulouse - Jean Jaurès. Elle assure son enseignement en LANSAD et en études filmiques. De 2009 à 2012, elle a été responsable de la cellule CLES et est actuellement responsable des UE de LANSAD niveaux B2 et C1.

Sa recherche au sein du laboratoire cultures anglo-saxonnes (CAS EA 801) porte sur l'évolution des représentations genrées (sens anglais de «gender ») et des relations de domination dans le cinéma hollywoodien. En parallèle, elle s'intéresse aux questions de transposition de la recherche en études filmiques aux contenus des enseignements en LANSAD et LLCE.

<cristelle.maury@univ-tlse2.fr> 